\title{
Special issue on IAPR MVA2013 best papers
}

\author{
Masaki Suwa $^{1} \cdot$ Yoichi Sato $^{2} \cdot$ Katsushi Ikeuchi $^{3}$
}

Published online: 4 November 2015

(C) Springer-Verlag Berlin Heidelberg 2015

With a number of successful deployments of various applications enabled by machine vision technologies in recent years, there is an increasing demand for machine vision applications to provide a key solution for emerging issues, with high impact on industry, society, and our everyday lives. In particular, machine vision applications are expected to play a fundamental role in solving important problems in safety, security, environmental, and industrial issues on a global scale. With the proliferation of camera networks consisting of low-cost/high-performance image sensors, and recent increases in computational power, the basic infrastructure is now readily available to support smart systems for various applications such as factory automation, surveillance, intelligent transport systems, and sensor networks for environmental monitoring. Our current challenge is to develop key machine vision technologies and systems which can meet practical solutions of such applications.

The 13th IAPR Conference on Machine Vision Applications, MVA2013, was held from May 20 through 23, 2013 at the Suzaku Campus, Ritsumeikan University, co-sponsored by the MVA Organization, IAPR TC-8, and Ritsumeikan University.

We received 156 submissions from 24 countries over the world. These submissions (32 papers for an oral session and 80 papers for a poster session) covered various solutions for emerging machine vision problems, and were selected through a rigorous peer-review performed by a strong Program Committee composed of active and up-and-coming researchers/engineers from around the world.

This special issue provides articles on state-of-the-art work on machine vision applications in the domains described above. Several high-grade papers, which received the best paper awards or highest scores in the review process, were selected by the guest editors. In addition, three papers extended from presentations at the MVA2013 were accepted in the special issue.

Finally, we would like to thank the reviewers for their evaluations, the authors for carefully revising their papers, as well as all those who contributed to the success of the original MVA 2013 meeting.
Masaki Suwa

suwa@ari.ncl.omron.co.jp

1 OMRON Corporation, Kyoto, Japan

2 The University of Tokyo, Tokyo, Japan

3 Microsoft Research Asia, Beijing, People's Republic of China 